\title{
Age-specific infectious period shapes dynamics of pneumonia in bighorn sheep
}

\author{
Authors: Raina K. Plowright, Kezia R. Manlove, Thomas E. \\ Besser, David J. Paez, Kimberly R. Andrews, Patrick E. \\ Matthews, Lisette P. Waits, Peter J. Hudson, and Frances \\ E. Cassirer
}

This is the peer reviewed version of the following article: see full citation below, which has been published in final form at https://dx.doi.org/10.1111/ele.12829. This article may be used for noncommercial purposes in accordance with Wiley Terms and Conditions for Self-Archiving.

Plowright, Raina K., Kezia R. Manlove, Thomas E. Besser, David J. Paez, Kimberly R. Andrews, Patrick E. Matthews, Lisette P. Waits, Peter J. Hudson, and Frances E. Cassirer. "Age-specific infectious period shapes dynamics of pneumonia in bighorn sheep." Ecology Letters 20, no. 10 (September 2017): 1325-1336. DOI: 10.1111/ele.12829.

Made available through Montana State University's ScholarWorks scholarworks. montana.edu 


\title{
Age-specific infectious period shapes dynamics of pneumonia in bighorn sheep
}

\author{
Raina K. Plowright, ${ }^{1}$ * \\ Kezia R. Manlove, ${ }^{2}$ \\ ${ }^{1}$ Department of Microbiology and Immunology, Montana State University, \\ 109 Lewis Hall, Bozeman, MT 59717, USA \\ Thomas E. Besser, ${ }^{2}$ David J. Páez, ${ }^{1}{ }^{2}$ Department of Veterinary Microbiology and Pathology, Washington State \\ Kimberly R. Andrews, ${ }^{3}$ \\ Patrick E. Matthews, ${ }^{4}$ \\ Lisette P. Waits, ${ }^{3}$ Peter J. Hudson ${ }^{5}$ \\ and E. Frances Cassirer ${ }^{6}$ \\ University, Pullman, WA 99164, USA \\ ${ }^{3}$ Department of Fish and Wildlife Sciences, University of Idaho, 875 \\ Perimeter Drive MS 1136, Moscow, ID 83844, USA \\ ${ }^{4}$ Oregon Department of Fish and Wildlife, 65495 Alder Slope Road, Enter- \\ prise, OR 97828, USA \\ ${ }^{5}$ Center for Infectious Disease Dynamics, 201, Life Sciences Building, \\ Pennsylvania State University, University Park, PA 16802, USA \\ ${ }^{6} / d a h o$ Department of Fish and Game, 3316 16th Street, Lewiston, ID \\ 83501, USA
}

\begin{abstract}
Superspreading, the phenomenon where a small proportion of individuals contribute disproportionately to new infections, has profound effects on disease dynamics. Superspreading can arise through variation in contacts, infectiousness or infectious periods. The latter has received little attention, yet it drives the dynamics of many diseases of critical public health, livestock health and conservation concern. Here, we present rare evidence of variation in infectious periods underlying a superspreading phenomenon in a free-ranging wildlife system. We detected persistent infections of Mycoplasma ovipneumoniae, the primary causative agent of pneumonia in bighorn sheep (Ovis canadensis), in a small number of older individuals that were homozygous at an immunologically relevant genetic locus. Interactions among age-structure, genetic composition and infectious periods may drive feedbacks in disease dynamics that determine the magnitude of population response to infection. Accordingly, variation in initial conditions may explain divergent population responses to infection that range from recovery to catastrophic decline and extirpation.
\end{abstract}

\section{INTRODUCTION}

A small proportion of the host population can be responsible for the majority of pathogen transmission (Woolhouse et al. 1997; Lloyd-Smith et al. 2005; Buhnerkempe et al. 2017). These individuals produce a disproportionate number of secondary infections by engaging in behaviour that increases transmission, shedding more pathogen per unit time, or remaining infected for longer than the average host (LloydSmith et al. 2005; Streicker et al. 2013; VanderWaal \& Ezenwa 2016). The consequences of superspreading were exemplified by the pandemics of severe acute respiratory syndrome (SARS) and human immunodeficiency virus (HIV) that were spread worldwide by a small number of highly infectious or connected individuals (May \& Anderson 1987; Lloyd-Smith et al. 2005). Since those landmark pandemics, the dynamic consequences of variation in infectiousness and social contact have been studied in detail (e.g., Ferrari et al. 2006; Bansal et al. 2007; Chase-Topping et al. 2008). By contrast, relatively little attention has focused on variation in infectious periods among individuals and the dynamic consequences of this heterogeneity (Woolhouse et al. 1997; Hawley \& Altizer 2011).

This knowledge gap is particularly relevant to management of many common pathogens of humans and animals that are persistently carried by some hosts. Persistently infected individuals help to maintain Salmonella, Brucella, Staphylococcus, Streptococcus, Mycobacterium, Haemophilus and Leptospira species, Hepatitis $\mathrm{B}$ and $\mathrm{C}$ virus, and Epstein-Barr virus within host populations (Medley et al. 2001; Young et al. 2002; Virgin et al. 2009; Prager et al. 2013; Buhnerkempe et al. 2017). Yet, for many if not most of these pathogens, the mechanisms driving persistence and prevalence are not understood (Wertheim et al. 2005). Such understanding comes from resampling individuals over time, but longitudinal empirical studies are expensive, difficult and rare (VanderWaal \& Ezenwa 2016). Moreover, inferences derived from cross-sectional studies can mislead control efforts. For example, longitudinal studies of Escherichia coli O157 in cattle, thought to be maintained by persistently infected supershedders, revealed that the pathogen may be serially shed by many individuals - in other words, different individuals take turns at supershedding (Spencer et al. 2015). If supershedding is not a stable individual-host characteristic, then targeted interventions based on specific individuals will fail.

Here, we investigate heterogeneity in infectious periods among hosts, and mechanisms that may give rise to heterogeneity, in a system where the pathogen is limiting host populations: Mycoplasma ovipneumoniae in bighorn sheep (Ovis canadensis) (Fig. 1). M. ovipneumoniae is the primary causal agent of bacterial pneumonia in bighorn sheep (Besser et al. 


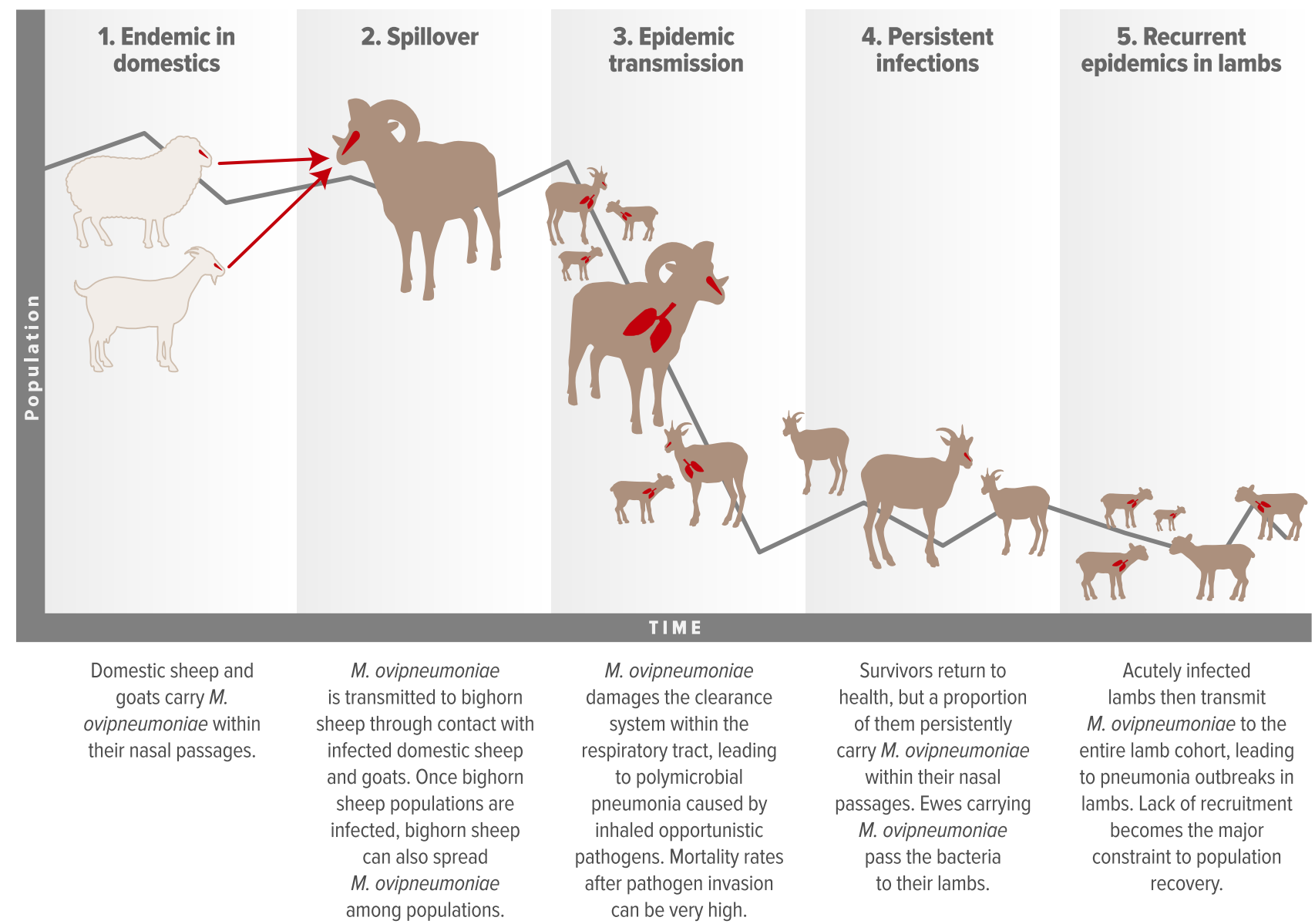

Figure 1 Epidemiology of Mycoplasma ovipneumoniae invasion and persistence in bighorn sheep populations.

2008, 2012a,b, 2013, 2014). This pathogen, likely introduced with domestic sheep during European settlement, contributed to the historic decline of bighorn sheep to less than 20000 individuals across the United States (Buechner 1960). Domestic sheep tolerate $M$. ovipneumoniae in their upper respiratory mucosa with varying morbidity but generally low mortality (Alley et al. 1999; Besser et al. 2012a). Introduction of $M$. ovipneumoniae into naïve bighorn sheep populations can result in severe mortality ranging from 10 to $100 \%$ (Cassirer et al. 2013, 2017). Animals that survive acute infection and associated disease can clear infection or can remain subclinically infected in the upper respiratory tract. If previously exposed animals are reinfected, they are relatively immune to lower respiratory tract disease but can develop a subclinical upper respiratory tract infection (Cassirer et al. 2013; Plowright et al. 2013). Infected populations often remain stagnant, or decline - sometimes to extirpation - in the years to decades after $M$. ovipneumoniae introduction because most lambs die of pneumonia by 3 months of age (Manlove et al. 2016). Sustained lack of recruitment is the primary impediment to bighorn sheep recovery.

We evaluated the support for two competing hypotheses about population-level persistence of $M$. ovipneumoniae in bighorn sheep. First, M. ovipneumoniae could be maintained by persistent infections within a few specific individuals. Second, $M$. ovipneumoniae could be maintained through serial (sequential) infections transmitted among many individuals. For the first hypothesis, the prediction is that infection status across sampling events would be positively correlated within individuals. In the second, the prediction is that an animal's infection status during one test would be independent of its status during previous tests, provided the intervals between testing are longer than the infectious period and that acquired immunity does not affect infection status. Moreover, the probability that an animal tested positive would reflect current population-level prevalence. Additionally, we investigated individual characteristics that might drive persistent carriage including age, genetic diversity and coinfection with lungworms. Finally, we predicted that lambs born to carrier ewes would be more likely to die than lambs from non-carriers because of early transmission directly from mother to offspring. To examine these hypotheses, we repeatedly sampled free-ranging bighorn sheep in the Lostine population (Wallowa Mountains, Eastern Oregon, USA) over a 4-year period.

\section{METHODS}

\section{Study population}

The Lostine bighorn sheep comprise a single, well-mixed population that has been surveyed annually since it was established in 1971 (see Supporting Information; Coggins 1988; 
Fig. 2). In 1986, a pneumonia epidemic killed two-thirds of the population; however, the population rebounded to $92 \%$ of its pre-epidemic size within 12 years (Coggins 1988; Coggins \& Matthews 1992; Cassirer et al. 2013). In 2000, a year before a novel $M$. ovipneumoniae strain was detected in the population (Cassirer et al. 2016), monitoring was expanded to include survival and productivity of individuals tracked with radio collars (Cassirer \& Sinclair 2007; Cassirer et al. 2013). Population estimates during this study ranged from 75 to 90 sheep (lambs and adults; ODFW, unpublished data; Fig. 2b).

\section{Sampling methods and diagnostic testing}

Bighorn sheep in the Lostine population migrate seasonally between low and high elevations (Coggins 1988) and are habituated to humans through supplemental feeding on their winter range. We used a baited corral trap and ground darting to capture animals on their winter range (Supporting Information). We placed unique markers on each animal and recaptured individuals within and between seasons with a bias towards sampling females because they transmit pathogens to lambs. We monitored 41 radio-collared animals during the study (34 females, 7 males) of which seven had been collared prior to the study.

We collected nasal samples with dacron swabs and placed these samples into mycoplasma broth (Hardy Diagnostics, Santa Maria, CA) for culture enrichment or into sterile tubes (dry swabs). We conducted PCR and RT-PCR (realtime PCR) detection of $M$. ovipneumoniae with the methods described in McAuliffe et al. (2003) and Ziegler et al. (2014), respectively. Both tests were used to ascribe infection status to a sample, and RT-PCR was also used to estimate relative pathogen load. We collected blood for detection of anti- $M$. ovipneumoniae antibodies by competitive enzyme-linked immunosorbent assay (ELISA) on serum (Besser et al. 2014). We collected faecal samples and measured lungworm larvae intensity with the Baermann technique (Forrester \& Lankester 1997). We also scored body condition by palpating the sacral crest (Cook et al. 2007). Diagnostic testing was conducted at the Washington Animal Disease Diagnostic Laboratory and in the Besser laboratory at Washington State University.

\section{Data analyses}

\section{Persistent carriage analysis}

We used a suite of non-parametric resampling-based methods to evaluate our hypotheses (Supporting Information). We compared the observed frequency of changes in animal infection status through time to that expected by chance. We repeatedly randomly permuted infection status for all individuals across all capture events. We calculated the average duration of an animal's infection status for each permutation and used those averages to build a null distribution reflecting the average time infected or not infected under the serial transmission hypothesis. We then compared the quantile of the null distribution to the observed average duration in each infection state to obtain a $P$ value for departure from the serial transmission hypothesis.

Moreover, we considered multiple tests of the same animal assuming a Hardy-Weinberg-like equilibrium (Wigginton et al. 2005). We considered a situation in which two tests of (a)

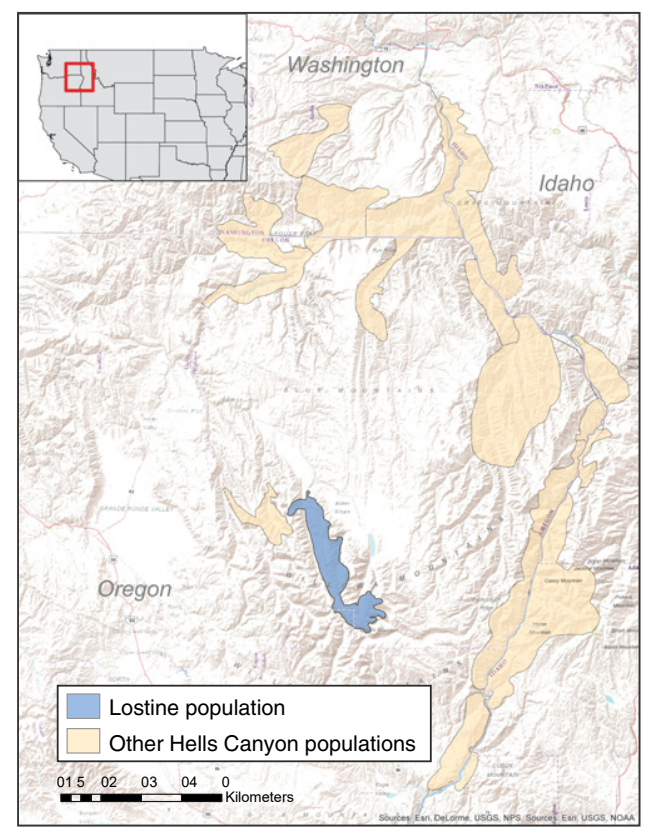

(b)

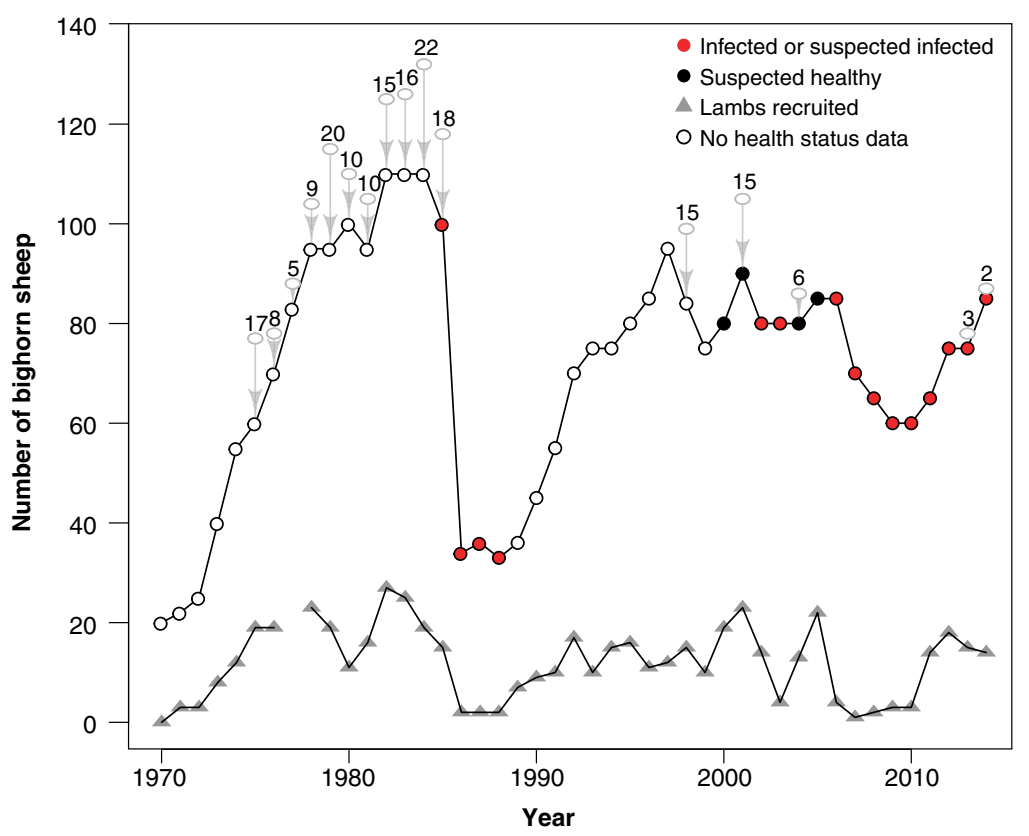

Figure 2 (a) Map of the Lostine Population. (b) Population trajectory and lamb recruitment of the Lostine population from 1970 to 2014 . Red dots indicate that Mycoplasma ovipneumoniae was detected by polymerase chain reaction in one or more individuals, or M. ovipneumoniae infection was suspected because pneumonic animals were observed, or juvenile survival through summer was less than $50 \%$. Black dots indicate that recruitment was high and we suspect that M. ovipneumoniae was not transmitted to lambs. Open circles indicate that no health data were available. Digits indicate the number of animals that were translocated out of the population and the length of the arrow corresponds to this number. 
the same individual were treated as analogous to two alleles, and we assumed the transmission and recovery processes were sufficiently rapid that consecutive test results for the same animal were independent (Supporting Information). Departures from an equilibrium pattern suggest that infection concentrates in a few hosts.

We created a frequency distribution of the proportion of positive test results for all animals tested three or more times. Each individual contributed one proportion to this distribution, and proportions were weighted according to the number of times animals were sampled, such that individuals with more samples received greater weight. Under the serial transmission hypothesis, the distribution would be unimodal and centred at the population prevalence; under the persistent carrier hypothesis it would be bimodal with individuals repeatedly testing either positive or negative. We fit a kernel density estimator over the range of this distribution (from 0 to 1 ), identified a natural breakpoint at the proportion corresponding to the minimum of the kernel density estimator and generated bootstrapped confidence intervals for the breakpoint (Supporting Information).

To explore the consistency of an individual's infection status across years, we estimated the probability that an animal had a positive test result in year $t+1$ given two or more positive test results in year $t$. We repeated this analysis for animals that always tested negative in the first year. We used binomial confidence intervals to quantify uncertainty in our estimates.

To account for the temporal pattern of infection status, we based our classification of individual animals as persistent carriers, intermittent carriers or negatives on the following definition: persistent carriers were individuals that tested positive in two or more consecutive sampling events across at least 2 years (even if they tested as negative at other times) and intermittent carriers tested both positive and negative in consecutive years. Animals that were only sampled once were not assigned a carriage status.

To explore whether anti-M. ovipneumoniae antibody levels and pathogen load (measured in RT-PCR cycle thresholds [Cts] on DNA extracted directly from nasal swab samples) differed among carriage classifications, we used Welch's twosample $t$-test.

\section{Age-specific infection status}

We assigned ages and birth years to bighorn sheep younger than 4 years old at initial capture $(n=67)$ on the basis of incisor eruption (Dimmick \& Pelton 1994). We assigned the ages of five animals 4 years or older when first captured on the basis of cementum analysis of incisors obtained at necropsy (Turner 1977; Mattson Laboratory, Milltown, MT).

For other animals that were 4 years of age or older at first capture, definitive age estimation was not possible $(n=20)$. We used a Monte Carlo simulation to estimate their ages. We defined a window of possible ages for each animal such that the minimum possible age at first capture was four and the maximum possible age at final capture was 20 (Nussey et al. 2008). Each time the animal was recaptured, we narrowed the lower and upper bound on this window. We drew the individual's estimated age at capture from this window with probabilities proportionate to age-specific survival estimates from Jorgenson et al. (1997) (Supporting Information).
We repeated the age estimation process for animals with unknown ages 10000 times and concatenated these ages to a fixed dataset of animals with known ages. At each iteration, we fit a mixed-effects logistic regression model (Bates et al. 2014) on the presence or absence of $M$. ovipneumoniae. The linear and quadratic effects of sheep age were included as fixed effects, whereas the animal's identity was modelled as a random effect. We regressed infection status on median age estimates, and used Akaike's information criteria (AIC) to compare models with linear and quadratic age. We also evaluated infection in cohorts of animals reconstructed from longitudinal demographic data (Supporting Information).

\section{Genetic diversity}

We extracted DNA from blood samples and then PCR-amplified and genotyped 15 autosomal DNA microsatellite loci and one sex chromosome locus for sex identification (Supporting Information and Table S1). Eleven of the autosomal loci were expected to be adaptively neutral given their position in the genome. The remaining four autosomal loci were located within (or close to) genes associated with immune system function (Supporting Information) and were putatively under disease-related adaptive pressures (e.g. Paterson et al. 1998b; Coltman et al. 2001; Luikart et al. 2008). We calculated neutral genetic diversity for each individual as the proportion of the 11 neutral loci that were heterozygous, and used Student's $t$-tests to evaluate whether carrier classes differed in the mean proportion of heterozygous genotypes across loci. For each putatively adaptive locus, we used Fisher's exact tests to evaluate whether the proportion of heterozygous individuals across carrier classes differed. We also incorporated heterozygosity at adaptive loci into the logistic regression model described above with each locus, age, and quadratic age included as covariates. We used AIC to compare models with and without heterozygosity (Supporting Information).

\section{Parentage, lamb survival and lamb infection status}

In 2012-2015, we assessed survival of offspring by observing marked ewes and lambs monthly in their remote habitat through summer. We classified a female as having a lamb if she was observed nursing a lamb, alone with a lamb or if she was pregnant the previous winter. We assumed she had lost her lamb if she was observed in a group with no lambs over several consecutive observations. In 2014 and 2015, we added genetic maternity analyses on blood and tissue samples from marked ewes and lambs and faecal samples from unmarked lambs, yearlings and ewes.

For all samples, we PCR-amplified the same 15 autosomal microsatellite and sex chromosome loci as described above, but with extra (3-6) independent PCR amplifications for faecal samples to account for potential allele dropout (Waits \& Paetkau 2005; Supporting Information). Duplicate samples from the same individual were genetically identified and removed as described in the Supporting Information.

We identified potential mothers as females $\geq 2$ years at the birth of the individual of interest. We used CERVUS 3.0.7 (Marshall et al. 1998; Kalinowski et al. 2007) to conduct likelihood assessment of maternity. We estimated that $90 \%$ of potential mothers in the population had been sampled, and 
required an $80 \%$ confidence level to assign maternities. We submitted serum for pregnancy testing (Drew et al. 2001) if a female sampled over the winter was never seen with a lamb in spring to determine whether she lost her lamb or was not pregnant.

We used a generalised mixed model with binomial errors and a logit link function to estimate whether offspring survival differed as a function of ewe carrier classification, or the results of the two closest tests preceding the lamb's birth (both positive, one positive or both negative). We included infection status and linear and quadratic ewe age as covariates and ewe identity as a random effect. We used AIC-based approaches for model selection.

\section{Lungworms}

Lungworm larvae counts were overdispersed and zeroinflated, hence we applied negative binomial models to these data with age, carriage status, infection status and heterozygosity at four adaptive loci as fixed effects. We also fit models with random effects for individual (Supporting Information).

\section{RESULTS}

\section{Infection status}

From April 2012 through March 2016, we captured and sampled 86 animals for a total of 227 samples. Forty percent $(N=34)$ of animals were sampled two to nine times (Table 1).

All adults, including infected individuals, were in good body condition throughout the study and most animals appeared healthy, although we occasionally saw signs of respiratory tract disease and associated weakness. Lamb-ewe ratios in late winter (when lambs are $\sim 9$ months old), and adult female survival, averaged $0.43(0.37-0.50)$ and $0.93(0.9-1)$, respectively (Supporting Information).

M. ovipneumoniae was detected in $47 \%$ of adult females (95\% bootstrapped confidence interval [35\%,65\%]), 44\% of yearlings $(11 \%, 78 \%)$ and $89 \%$ of lambs $(78 \%, 100 \%)$ (Fig. 3a). Antibodies to M. ovipneumoniae (at $\geq 50 \%$ inhibition) were detected at least once in $95 \%$ (41/43) of adult females, $85 \%$ of yearlings (17/20) and 97\% of lambs (31/32).

Antibody levels from animals that tested PCR-positive were significantly higher than those from animals that tested

Table 1 Number of bighorn sheep sampled between 2012 and 2016, and number of times sampled, by sex and age*

\begin{tabular}{lrrrrrrrrrr}
\hline Number of times sampled & 1 & 2 & 3 & 4 & 5 & 6 & 7 & 8 & 9 & Total \\
\hline Total individuals & 52 & 6 & 3 & 6 & 5 & 11 & 2 & 0 & 1 & 86 \\
Adult ewes & 12 & 5 & 3 & 6 & 5 & 11 & 2 & 0 & 1 & 45 \\
Adult rams & 5 & 1 & 0 & 0 & 0 & 0 & 0 & 0 & 0 & 6 \\
Yearlings* & 14 & 2 & 0 & 0 & 0 & 0 & 0 & 0 & 0 & 16 \\
Lambs* & 31 & 1 & 0 & 0 & 0 & 0 & 0 & 0 & 0 & 32 \\
Total samples & 62 & 18 & 9 & 24 & 25 & 66 & 14 & 0 & 9 & 227 \\
\hline
\end{tabular}

*Animals 2 years or older are classified as adults. Three lambs and six yearlings were sampled again as adults, and are included in the table twice. Two animals were sampled as lambs, yearlings and adults and are included in the table three times. negative on PCR on the same sampling event (Kruskal-Wallis test, $P<0.001, N=114$ PCR positives and 111 PCR negatives; Fig. S1). Antibody levels and pathogen loads in intermittent carriers (when infected) did not differ significantly from those in infected persistent carriers (Wilcoxon rank-sum $W=534.5, P=0.13$; Wilcoxon rank-sum $W=32, P=0.96$, respectively, Supporting Information).

\section{Persistent carriage analysis}

During our 4-year sampling period, the longest observation of consistently positive and negative test results from an individual animal were 3.2 and 3.1 years, respectively. However, the true duration of the same infection status could be longer; 44 of 50 infection events detected in adults in this study were either interval or right-censored (Fig. 4a).

Both the permutation analysis and the Hardy-Weinberg equilibrium analysis suggested that the average duration of positive or negative states was greater than expected by chance $\left(\mathrm{H}-\mathrm{W} \quad \mathrm{p}^{2}=0.14 ; \quad \mathrm{q}^{2}=0.39 ; \quad 2 \mathrm{pq}=0.47 ; \quad P\right.$-value $<$ 0.001 ; observed average exceeded all 5000 permutations for average duration in either state and for duration of the negative state; observed average exceeded all but 2 of 5000 permutations for the infected state; Fig. 3e-g; Fig. S2).

The kernel density approach suggested that animals separated into two groups when the proportion of positive tests was above or below $65.7 \%$ (95\% bootstrapped CI for the separation threshold $=[38 \%, 85 \%])($ Fig. 3c). Eight of $11(73 \%)$ animals that tested positive twice in year $t$ and were sampled at least once in year $t+1$ tested positive at least once in $t+1$ (95\% CI from 39 to $93 \%$ ). Seven of 10 animals that tested negative at least twice in year $t(70 \%)$, did not test positive in year $t+1(95 \%$ CI from 35 to $92 \%)$.

The final classifications of individuals that were sampled more than once are presented in Fig. 4a. Thirty-seven percent of animals were consistently positive over two or more years and were classified as persistent carriers. Forty-five percent of animals were both positive and negative, and were classified as intermittent carriers. The remaining $18 \%$ of animals had no PCR-positive test results, and were classified as negative (Figs $3 b$ and $4 a$ ).

\section{Age-specific infection status}

We found a significant, U-shaped quadratic relation between age and $M$. ovipneumoniae prevalence such that prevalence of infection was higher in relatively young ( $<2$ years) and old ( $>15$ years) individuals than in intermediate-aged animals (Fig. 5a). The AIC of a model that included both quadratic and linear terms was less than that of a model that included only a linear term $(\triangle \mathrm{AIC}=32$; Table $\mathrm{S} 2)$. Results from the cohort assignment ageing method were similar (Fig. 5b, Fig. S3).

\section{Genetic diversity and infection status}

Diversity at a putatively adaptive locus differed across carrier classes, whereas neutral genetic diversity did not (Fig. S4). A locus within the Major Histocompatibility I gene complex 
(MHCI) had lower heterozygosity for persistent carriers than intermittent carriers $(P=0.005)$ or negative animals $(P=0.04)$ (Fig. 3d). However, diversity at the other three putatively adaptive loci was not correlated with carrier status. In a model with mean age, but no random effect for individual identity (mixed models did not converge), animals that were
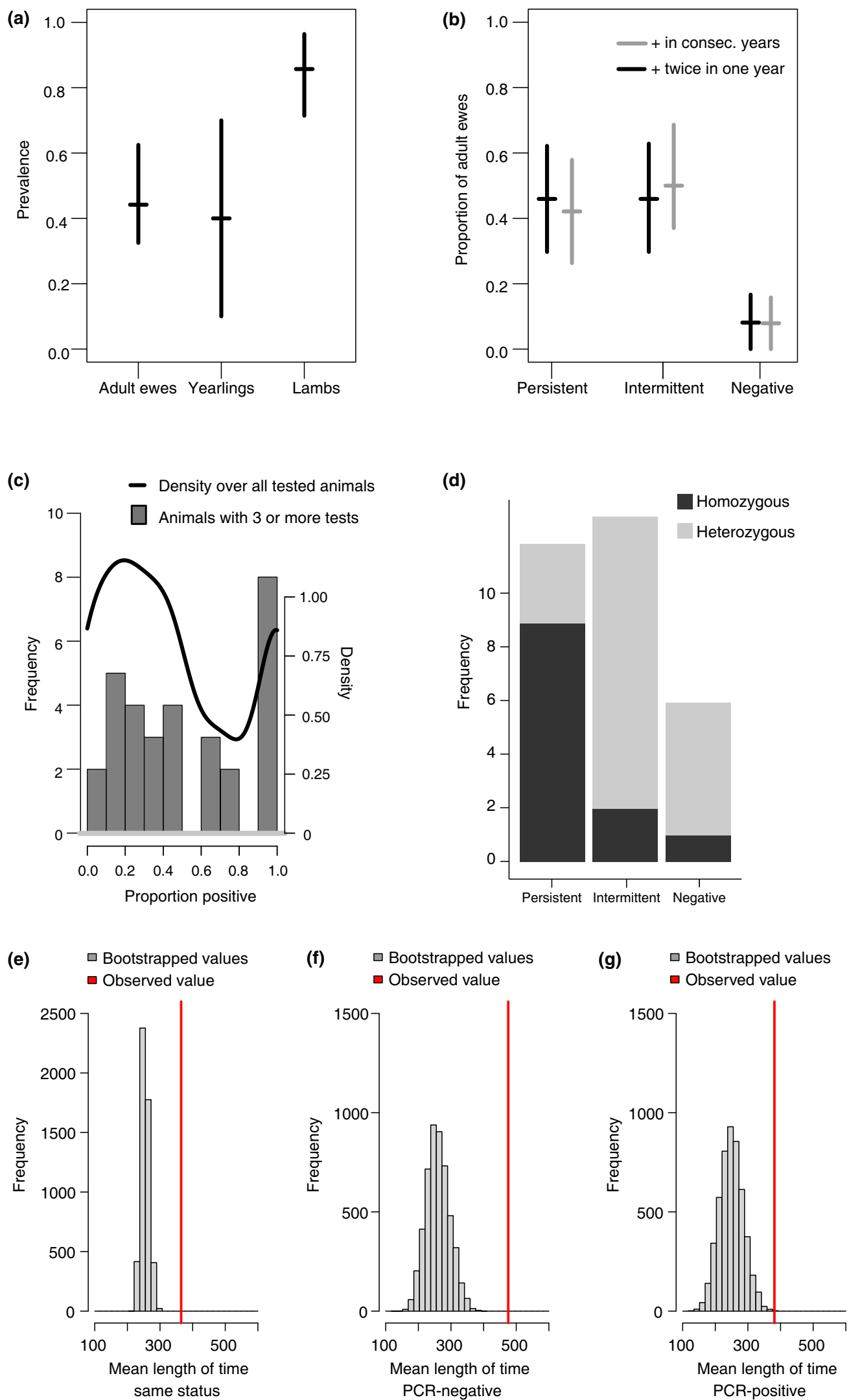
Figure 3 (a) Mean prevalence (with 95\% confidence intervals) of Mycoplasma ovipneumoniae shedding in adult females, yearlings and lambs. (b) Proportion of adult ewes characterised as persistent carriers, intermittent carriers or negative, given that M. ovipneumoniae was detected in consecutive years (light grey) or was detected at least twice in 1 year (dark grey). (c) The proportion of positive tests for 30 adult females tested three or more times in the Lostine bighorn sheep population, 2012-2016. The density curve is weighted by the number of tests. (d) Distribution of homozygosity and heterozygosity at a locus within the Major Histocompatibility I gene complex among three classes of $M$. ovipneumoniae carriage. (e, f, g) The expected and observed distributions of consecutive positive and negative tests. The average duration of both infected and uninfected states was greater than expected by chance by both a permutation analysis (e, f, g) and an equilibrium analysis (Fig. S2).

heterozygous at MHCI were less likely to be infected than animals that were homozygous at this locus $(P=0.016)$; age and quadratic age were both significant $(P<0.0001$; Table S3). However, models excluding and including all four adaptive loci had equivalent AIC scores (Supporting Information).

\section{Parentage, lamb survival and lamb infection status}

After removing duplicate samples, we identified 15 individuals from 97 faecal samples with final consensus genotypes. Genetic maternity analyses assigned 36 of 37 lambs to a dam, with 25 assignments at the 95\% confidence level and 11 assignments at the $80 \%$ confidence level.

A greater proportion of lambs died from ewes classified as persistent carriers compared to lambs from ewes classified as intermittent carriers or negative (Fig. S5). However, this result was not statistically significant $(P=0.45)$. When defining the carrier status of ewes based on the two tests prior to lambing, the difference in the survival of offspring of persistent and intermittent carrier ewes vs. negative ewes was non-significant $(P=0.09)$ (Fig. S5). Ewe age (linear or quadratic) was not associated with offspring survival (Supporting Information).
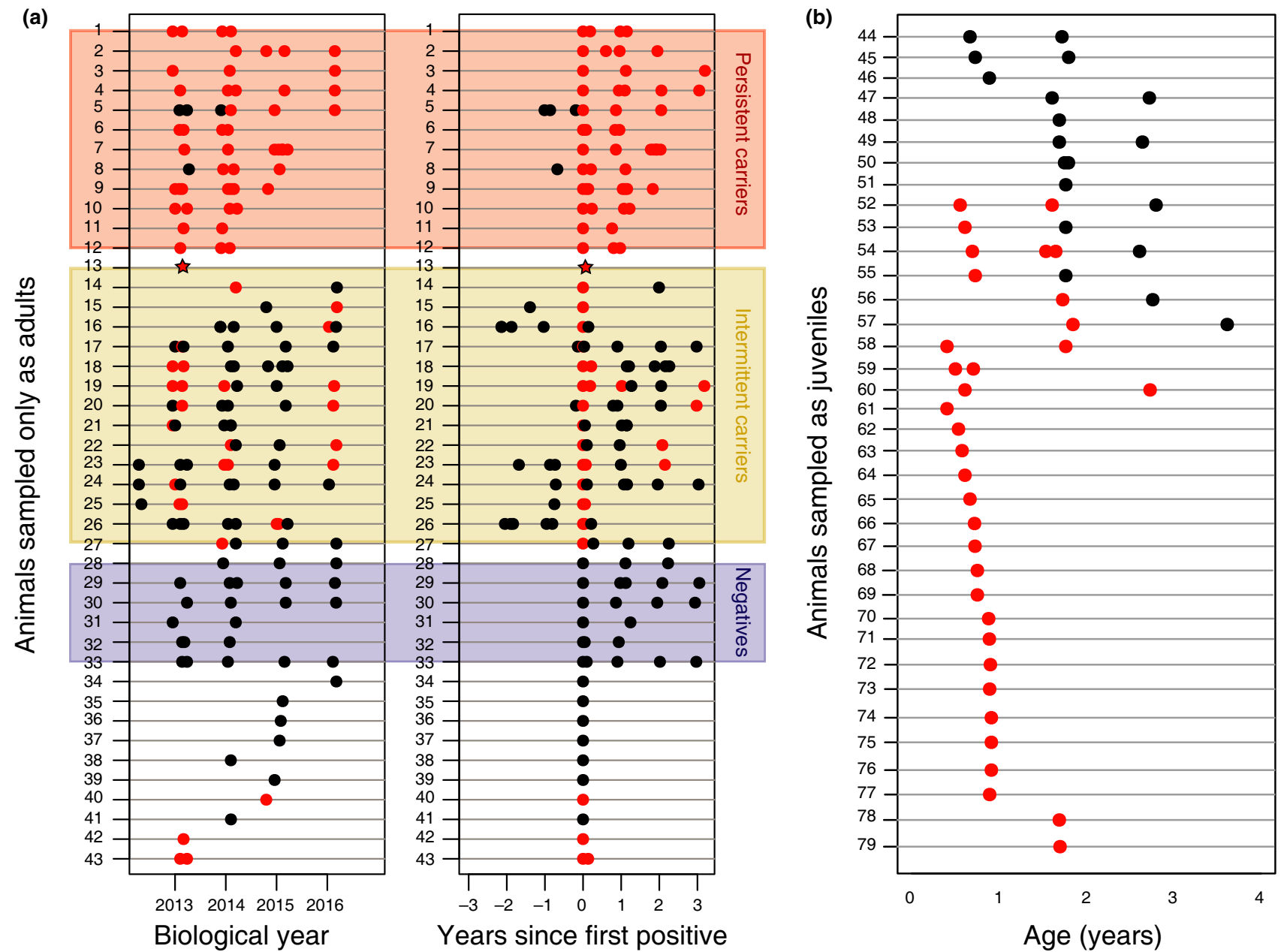

Figure 4 (a) Infection status of adult bighorn sheep over time. Red dots represent a positive test, black dots represent a negative test, based on PCR. Three classes were detected for animals captured two or more times: persistent carriers, intermittent carriers and negative. The star denotes an animal that was classified in part on samples (and PCR tests) collected prior to this study (Animal 13). All animals except 61, 41 and 6 were adult females. Biological year is 1 May to 31 April. (b) Infection status of bighorn sheep first sampled as juveniles (lambs or yearlings). 

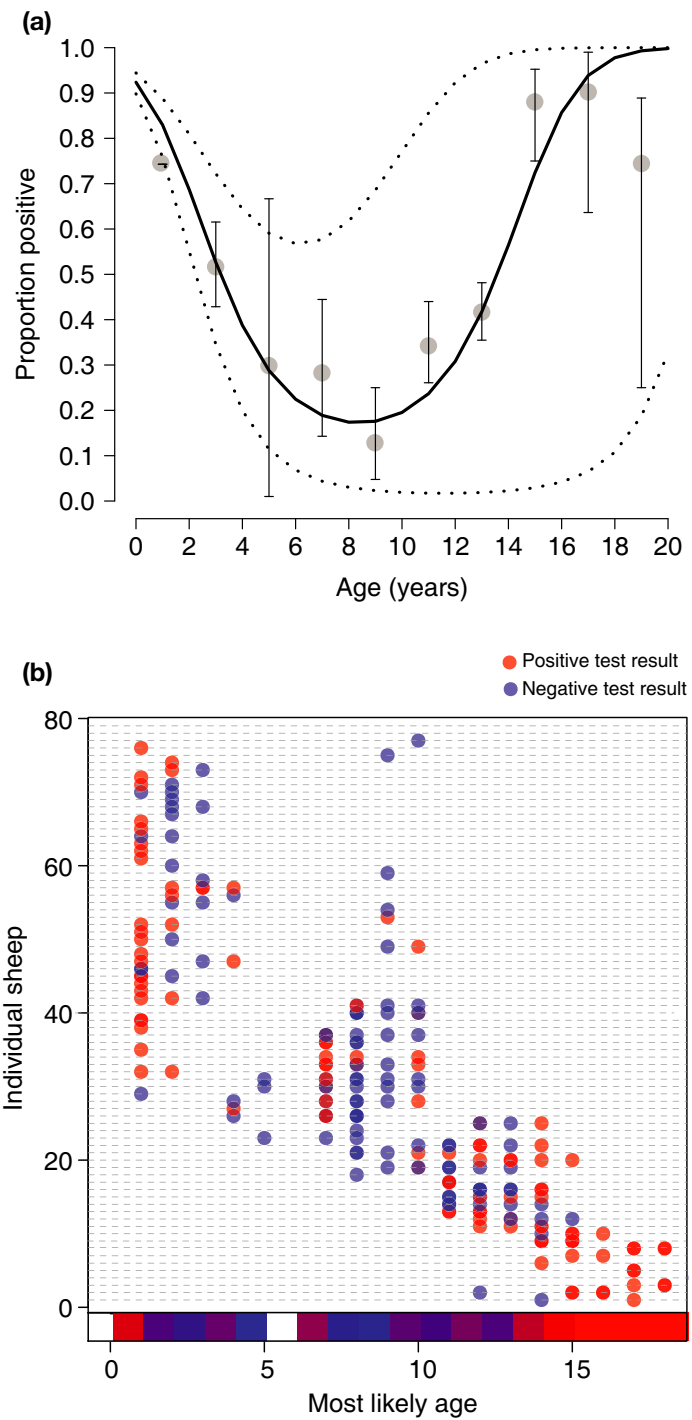

Figure 5 Relations between Mycoplasma ovipneumoniae prevalence and bighorn sheep age. (a) Error bars represent 95\% confidence intervals. The solid line is the fitted result from a generalised mixed-effects model with a logit link function and binomial errors, whereas the dotted lines are the $95 \%$ confidence intervals estimated by bootstrapping. (b) Age estimated by assigning animals to cohorts on the basis of sampling history, agespecific ewe survival estimates and annual recruitment data.

Of the 12 lambs or yearlings sampled over multiple years, four were negative and remained negative the following year; eight were positive and six of these lambs or yearlings became negative within 2 years (Fig. 4b).

Both ageing and carriage status (but not genetics, or infection status) competed to explain variation in lungworm counts, however, evidence of confounding made it difficult to establish causal links. Age probably underlies both carriage and lungworm status (Table S4, Fig. S6, Supporting Information).

\section{DISCUSSION}

Mycoplasma ovipneumoniae prevalence was highest in relatively young and old animals. However, the majority of infected young animals recovered within 1 to 2 years, whereas the majority of infected old animals remained infected for the duration of the study. The presence of older persistent carriers supports the hypothesis that $M$. ovipneumoniae is maintained by persistent infections within a few individuals, although, a high proportion of animals were intermittently infected and their contribution to pathogen persistence at the population level is unknown. If intermittent carriers sustain serial transmission without reinfection from persistent carriers, or if intermittent carriers are persistently infected with low pathogen loads that are only intermittently detected, removal of both intermittent and persistent carriers may be necessary to eliminate $M$. ovipneumoniae from populations.

Age-related responses to microparasitic and macroparasitic infections are well documented and are usually attributed to dynamic interactions among exposure, parasite-induced host mortality, parasite accumulation, acquired immunity, and agerelated variation in immune function (Hudson \& Dobson 1995; Wilson et al. 2002). The early peak in pathogen prevalence in bighorn sheep lambs may reflect early exposure of lambs to infected ewes, lack of protection by maternal immunity, and intense transmission among lambs concentrated in nursery groups. The high prevalence in older animals may be influenced by seasonal re-exposure during epidemics of pneumonia among lambs. However, given that evidence of exposure (by antibody detection) was also high in middle-aged animals that tested negative, low resistance to infection in young and old animals seems a more parsimonious explanation for the U-shaped relations between age and infection in bighorn sheep.

High risk of infection or mortality in the young and the old has been attributed to immune system immaturity and senescence, respectively (Lloyd 1995; Vallejo 2007). For example, children have the highest rates of infection with the persistent, upper respiratory tract pathogens Staphylococcus aureus and Kingella kingae; then, as the immune system matures, children either clear the pathogens or become intermittently infected (Wertheim et al. 2005; Yagupsky 2015). Higher risk in older animals is often attributed to immunosenescence and waning of immune memory. As individuals age, telomere lengths in immune cells erode, triggering replicative senescence of those cells (Vallejo 2007). The result is reduced activation, memory and responsiveness of the adaptive and innate immune systems (Tarazona et al. 2002; Solana et al. 2006). Declines in immunity as age increases explain increasing intensities of gastrointestinal helminths in Soay sheep (Ovis aries) (Hayward et al. 2009) and progression of bovine tuberculosis in badgers (Meles meles) (Beirne et al. 2016). Moreover, as exemplified by avian malaria (Asghar et al. 2015), long-term persistent infections can exacerbate telomere degradation and immunosenescence.

A combination of the mechanisms outlined above may explain the U-shaped pattern observed in this study. Similar combinations of mechanisms were suggested to explain the Ushaped relation between age and prevalence of human papillomavirus (HPV) infection and between age and mortality from influenza and varicella-zoster virus (Castle et al. 2005; Heininger \& Seward 2006; Greer et al. 2010). U-shaped age-infection curves are infrequently reported; age-intensity or age-prevalence curves that are convex or rise to an asymptote are more 
common and are a consequence of immunity or parasiteinduced mortality. Examples include lungworm larvae burdens observed in this study (Table S4) and Trichostrongylus retortaeformis in wild European rabbits (Oryctolagus cuniculus) (Cornell et al. 2008).

Despite many studies of age-varying risk of infection, few studies report age-varying duration of infection, probably because resampling individuals with known ages is seldom possible. However, there are rare exceptions. In humans, the probability of persistent Hepatitis B infection decreased with age (Ott et al. 2012), whereas, in some populations, the probability of persistent HPV infection increased with age (Castle et al. 2005). In cattle, the probability of persistent foot-andmouth disease virus infection decreased with age (Bronsvoort et al. 2016), however in wild rodents, no association was observed between the duration of cowpox infection and age (Hazel et al. 2000).

The interaction between age-structured carriage and lack of recruitment may explain why bighorn sheep population responses to $M$. ovipneumoniae infection range from recovery to catastrophic decline and extirpation. One population-level consequence of low recruitment is a shift towards an older age structure (Plowright et al. 2013; Manlove et al. 2016). If older animals are more likely to carry $M$. ovipneumoniae, then positive feedbacks may determine the prevalence in a population and the probability of the population's long-term persistence. For example, increasing mean age may lead to increasing prevalence of $M$. ovipneumoniae, increasing probability and severity of lamb pneumonia outbreaks and accelerating long-term population declines. Conversely, decreasing mean age may facilitate pathogen extinction and population recovery. Therefore, the age-structure during and immediately after pathogen-invasion could be important in determining the long-term population response to infection. Moreover, interventions to remove carriers may be most efficiently conducted after the initial outbreak and before the mean age increases as a consequence of lack of recruitment. These dynamic aspects of carriage are relatively unexplored. Nevertheless, disentangling these dynamic interactions is a challenging and necessary step towards understanding control options and long-term sequelae of infection.

In addition to age, genetic diversity at a locus within the MHCI gene complex was lower in persistent carriers than in negative or intermittent carriers. These results remained significant in a model including age. However, there was no relation between neutral genetic diversity and carrier status, suggesting that an individual's genotype at certain loci may influence infection status, whereas an individual's relative level of inbreeding does not. MHC class I alleles usually respond to intracellular pathogens and the mechanisms by which they would affect the presumably extracellular $M$. ovipneumoniae are unknown. However, MHC class I and II genes were linked in Soay sheep; if also true for this population, selective pressures at any MHC gene could generate genetic signatures across all MHC genes, including class II genes that respond to extracellular pathogens (Paterson 1998). MHC alleles in Soay and Scottish Blackface sheep were associated with survival and resistance to macroparasites (Schwaiger et al. 1995; Buitkamp et al. 1996; Paterson et al. 1998a).
The abundance of MHCI homozygous genotypes in older animals (Fig. S7) may reflect recent introduction of new genotypes through immigration, and may confound the age-carriage relations. Alternatively, homozygous and heterozygous individuals may have a survival advantage, or disadvantage, respectively. Counterintuitively, homozygotes could live longer than heterozygotes if homozygotes are tolerant (maintaining health despite their pathogen load) and heterozygotes are resistant but at a cost (e.g. energetic cost of immunity or immunopathology) (Raberg et al. 2009). In theory, selection for tolerant homozygotes could lead to a stable association between host and pathogen (Roy \& Kirchner 2000). However, for bighorn sheep, the phenomenon could be maladaptive because lambs would be susceptible to infections from tolerant adults. Thus, relations among genetic diversity, survival, tolerance and resistance, could drive another feedback loop that results in population decline. More data are needed to examine this hypothesis.

We found little evidence that a lamb's survival is directly related to its mother's infection status. Instead, the offspring of both carriers and non-carriers died, probably because lamb-to-lamb transmission dominates when lambs are concentrated in nursery groups, concealing the origin of the epidemic (Cassirer et al. 2013; Plowright et al. 2013; Manlove et al. 2014). Some offspring of persistent carriers survived, despite their putative early exposure, and offspring of non-carriers were exposed to other infected lambs or ewes. Lambs and adults that were PCR negative had serological evidence of exposure to $M$. ovipneumoniae, indicating that their status was not due to lack of exposure.

\section{Priorities for future research}

Our work highlights some critical knowledge gaps in understanding the population dynamics of persistently carried pathogens. First, there is a dearth of age-structured longitudinal data on infections in natural systems. Without data on age, potential mechanisms and feedbacks driving disease dynamics may be overlooked or misinterpreted (Medley et al. 2001). For example, one might mistakenly conclude that $M$. ovipneumoniae status drives lungworm burdens, whereas age probably drives both lungworm burden and $M$. ovipneumoniae status.

Second, there is little understanding of the role of intermittent carriers in maintaining and transmitting infections (Plowright et al. 2016). Experiments in the field and on captive animals, supported by modelling studies, may be necessary to clarify the role of intermittent carriers. Ultimately, replicated field experiments that monitor the outcome of removal of persistent or intermittent carriers may be needed to elucidate mechanisms of persistence at the population level.

Third, there is no robust statistical definition of persistent carriage, even for common human pathogens such as $S$. aureus (Wertheim et al. 2005). Our definition of persistent carriage within an individual as two consecutive years of infection was subjective given the constraints imposed by censoring, lack of data and limited knowledge of the relevant mechanisms. Longer studies are needed to empirically establish this definition in this system and others. Ideally, a 
definition informed by the number of secondary infections produced by individuals, as was developed for superspreaders (Lloyd-Smith et al. 2005), could be developed for persistent carriage. However, quantifying transmission of mucosal bacterial pathogens remains very challenging.

For bighorn sheep, we suggest that replicate studies be conducted in other locations to determine if the Lostine population - with its winter aggregation and particular strain of $M$. ovipneumoniae - is representative of other infected bighorn sheep populations. In addition, longer time series would allow stronger inference about age-prevalence relations and exploration of alternative explanations such as cohort effects (Supporting Information). The discovery of age-structured persistent carriage and variation in diversity at an MHC locus provides a basis for simulations and field experiments that explore options for eradication of pneumonia in bighorn sheep populations.

\section{ACKNOWLEDGEMENTS}

Thanks to Vic Coggins for his years of dedicated bighorn sheep management that made this study possible. Thanks to Holly E. Tuers-Lance and Chad Dotson for their assistance in the field and Nathan Justice, Alan Swanson, Jordan Schupbach and Robyn Egloff for guidance on analyses or figures. Funding was provided by Morris Animal Foundation grant D13ZO-081, National Institutes of Health IDeA Program grants P20GM103474 and P30GM110732, P. Thye, Montana University System Research Initiative: 51040-MUSRI2015-03, Oregon Chapter of the Foundation for North American Wild Sheep, Idaho Department of Fish and Game, Washington Department of Fish and Wildlife, Oregon Department of Fish and Wildlife, Federal Aid to Wildlife Restoration, and University of Idaho. KRM was supported on a Pennsylvania State University Academic Computing Fellowship.

\section{AUTHORSHIP}

RKP and EFC designed the study; PEM, EFC and RKP supported field data collection; TEB contributed to diagnostic testing; KRA and LPW performed genetic analyses; KM, RKP, DP and EFC analysed data; RKP, EFC and KM wrote the first draft of the manuscript and all authors contributed to revisions.

\section{DATA ACCESSIBILITY STATEMENT}

Data are available from the Dryad Digital Repository: http:// dx.doi.org/10.5061/dryad.s86sd.

\section{REFERENCES}

Alley, M., Ionas, G. \& Clarke, J. (1999). Chronic non-progressive pneumonia of sheep in New Zealand-a review of the role of Mycoplasma ovipneumoniae. N. Z. Vet. J., 47, 155-160.

Asghar, M., Hasselquist, D., Hansson, B., Zehtindjiev, P., Westerdahl, H. $\&$ Bensch, S. (2015). Hidden costs of infection: chronic malaria accelerates telomere degradation and senescence in wild birds. Science, $347,436-438$.
Bansal, S., Grenfell, B.T. \& Meyers, L.A. (2007). When individual behaviour matters: homogeneous and network models in epidemiology. J. R. Soc. Interface, 4, 879-891.

Bates, D., Mächler, M., Bolker, B. \& Walker, S. (2014). Fitting linear mixed-effects models using lme4. arXiv preprint arXiv:1406.5823.

Beirne, C., Waring, L., McDonald, R.A., Delahay, R. \& Young, A. (2016). Age-related declines in immune response in a wild mammal are unrelated to immune cell telomere length. Proc. R. Soc. London B: Biol. Sci., 283.

Besser, T.E., Cassirer, E.F., Potter, K.A., VanderSchalie, J., Fischer, A., Knowles, D.P. et al. (2008). Association of Mycoplasma ovipneumoniae infection with population-limiting respiratory disease in free-ranging Rocky Mountain bighorn sheep (Ovis canadensis canadensis). J. Clin. Microbiol., 46, 423-430.

Besser, T.E., Cassirer, E.F., Yamada, C., Potter, K.A., Herndon, C.N., Foreyt, W.J. et al. (2012a). Survival of bighorn sheep (Ovis canadensis) commingled with domestic sheep (Ovis aries) in the absence of Mycoplasma ovipneumoniae. J. Wildl. Dis., 48, 168-172.

Besser, T.E., Highland, M.A., Baker, K., Cassirer, E.F., Anderson, N.J., Ramsey, J.M. et al. (2012b). Causes of pneumonia epizootics among Bighorn Sheep, Western United States, 2008-2010. Emerg. Infect. Dis., 18, 406-414.

Besser, T.E., Frances Cassirer, E., Highland, M.A., Wolff, P., JusticeAllen, A., Mansfield, K. et al. (2013). Bighorn sheep pneumonia: sorting out the cause of a polymicrobial disease. Prev. Vet. Med., 108, 85-93.

Besser, T.E., Cassirer, E.F., Potter, K.A., Lahmers, K., Oaks, J.L., Shanthalingam, S. et al. (2014). Epizootic pneumonia of bighorn sheep following experimental exposure to Mycoplasma ovipneumoniae. PLoS ONE, 9, e110039.

Bronsvoort, B.M.d., Handel, I.G., Nfon, C.K., Sørensen, K.-J., Malirat, V., Bergmann, I. et al. (2016). Redefining the 'carrier' state for footand-mouth disease from the dynamics of virus persistence in endemically affected cattle populations. Sci. Rep., 6, 29059.

Buechner, H.K. (1960). The bighorn sheep in the United States, its past, present, and future. Wildl. Monogr., 4, 3-174.

Buhnerkempe, M.G., Prager, K.C., Strelioff, C.C., Greig, D.J., Laake, J.L., Melin, S.R., et al. (2017). Detecting signals of chronic shedding to explain pathogen persistence: Leptospira interrogans in California sea lions. J Anim Ecol., 86, 460-472.

Buitkamp, J., Filmether, P., Stear, M.J. \& Epplen, J.T. (1996). Class I and class II major histocompatibility complex alleles are associated with faecal egg counts following natural, predominantly Ostertagia circumcincta infection. Parasitol. Res., 82, 693-696.

Cassirer, E.F. \& Sinclair, A.R.E. (2007). Dynamics of pneumonia in a bighorn sheep metapopulation. J. Wildl. Manage., 71, 1080-1088.

Cassirer, E.F., Plowright, R.K., Manlove, K.R., Cross, P.C., Dobson, A.P., Potter, K.A. et al. (2013). Spatio-temporal dynamics of pneumonia in bighorn sheep. J. Anim. Ecol., 82, 518-528.

Cassirer, E.F., Manlove, K.R., Plowright, R.K. \& Besser, T.E. (2016). Evidence for strain-specific immunity to pneumonia in bighorn sheep. J. Wildl. Manage., 81, 133-143.

Cassirer, E. F., Manlove, K. R., Almberg, E. S., Kamath, P. L., Cox, M., Wolff, P., et al. (2017), Pneumonia in bighorn sheep: Risk and resilience. J. Wild. Manage. https://doi.org/10.1002/jwmg.21309

Castle, P.E., Schiffman, M., Herrero, R., Hildesheim, A., Rodriguez, A.C., Bratti, M.C. et al. (2005). A prospective study of age trends in cervical human papillomavirus acquisition and persistence in Guanacaste, Costa Rica. J. Infect. Dis., 191, 1808-1816.

Chase-Topping, M., Gally, D., Low, C., Matthews, L. \& Woolhouse, M. (2008). Super-shedding and the link between human infection and livestock carriage of Escherichia coli O157. Nat. Rev. Microbiol., 6, 904-912.

Coggins, V.L. 1988. The Lostine Rocky Mountain bighorn sheep die-off and domestic sheep. In Proceedings of the 6th Biennial Symposium of the Northern Wild Sheep and Goat Council (ed Baniff W. M.). Banff, Alta., 11-15 April 1988. pp.57-64. 
Coggins, V. L. \& Matthews, P. E. (1992). Lamb survival and herd status of the Lostine bighorn herd following a Pasteurella die-off. In Proceedings of the Biennial Symposium of the Northern Wild Sheep and Goat Council, (eds Emmerich J., Hepworth, W.G.). Cody, WY, 8:147154.

Coltman, D., Wilson, K., Pilkington, J., Stear, M. \& Pemberton, J. (2001). A microsatellite polymorphism in the gamma interferon gene is associated with resistance to gastrointestinal nematodes in a naturallyparasitized population of Soay sheep. Parasitology, 122, 571-582.

Cook, R.C., Stephenson, T.R., Myers, W.L., Cook, J.G. \& Shipley, L.A. (2007). Validating predictive models of nutritional condition for mule deer. J. Wildl. Manage., 71, 1934-1943.

Cornell, S.J., Bjornstad, O.N., Cattadori, I.M., Boag, B. \& Hudson, P.J. (2008). Seasonality, cohort-dependence and the development of immunity in a natural host-nematode system. Proc. Biol. Sci., 275, 511-518.

Dimmick, R.W. \& Pelton, M.R. (1994). Criteria of sex and age. In: Research and Management Techniques for Wildlife and Habitats, 5th edn. The Wildlife Society, Bethesda, MA, pp. 169-214.

Drew, M.L., Bleich, V.C., Torres, S.G. \& Sasser, R.G. (2001). Early pregnancy detection in mountain sheep using a pregnancy-specific protein B assay. Wildl. Soc. Bull., 29, 1182-1185.

Ferrari, M.J., Bansal, S., Meyers, L.A. \& Bjørnstad, O.N. (2006). Network frailty and the geometry of herd immunity. Proc. Biol. Sci., 273, 2743-2748.

Forrester, S.G. \& Lankester, M.W. (1997). Extracting Protostrongylus spp. larvae from bighorn sheep feces. J. Wildl. Dis., 33, 868-872.

Greer, A., Tuite, A. \& Fisman, D. (2010). Age, influenza pandemics and disease dynamics. Epidemiol. Infect., 138, 1542-1549.

Hawley, D. \& Altizer, S.M. (2011). Disease ecology meets ecological immunology: understanding the links between organismal immunity and infection dynamics in natural populations. Funct. Ecol., 25, 48-60.

Hayward, A.D., Wilson, A.J., Pilkington, J.G., Pemberton, J.M. \& Kruuk, L.E. (2009). Ageing in a variable habitat: environmental stress affects senescence in parasite resistance in St Kilda Soay sheep. Proc. Biol. Sci., 276, 3477-3485.

Hazel, S., Bennett, M., Chantrey, J., Bown, K., Cavanagh, R., Jones, T. et al. (2000). A longitudinal study of an endemic disease in its wildlife reservoir: cowpox and wild rodents. Epidemiol. Infect., 124, $551-562$.

Heininger, U. \& Seward, J.F. (2006). Varicella. Lancet, 368, 1365-1376.

Hudson, P. \& Dobson, A. (1995). Macroparasites: observed patterns in naturally fluctuating animal populations. In: Ecology of Infectious Diseases in Natural Populations (eds. Grenfell, B.T. and Dobson, A.P.). Cambridge University Press, Cambridge, pp. 144-176.

Jorgenson, J.T., Festa-Bianchet, M., Gaillard, J.-M. \& Wishart, W.D. (1997). Effects of age, sex, disease, and density on survival of bighorn sheep. Ecology, 78, 1019-1032.

Kalinowski, S.T., Taper, M.L. \& Marshall, T.C. (2007). Revising how the computer program CERVUS accommodates genotyping error increases success in paternity assignment. Mol. Ecol., 16, 1099-1106.

Lloyd, S. (1995). Environmental Influences on Host Immunity. Ecology of Infectious Diseases in Natural Pop-ulations. Cambridge University Press, Cambridge, UK, pp. 327-361.

Lloyd-Smith, J.O., Schreiber, S.J., Kopp, P.E. \& Getz, W.M. (2005). Superspreading and the effect of individual variation on disease emergence. Nature, 438, 355-359.

Luikart, G., Pilgrim, K., Visty, J., Ezenwa, V.O. \& Schwartz, M.K. (2008). Candidate gene microsatellite variation is associated with parasitism in wild bighorn sheep. Biol. Let., 4, 228-231.

Manlove, K.R., Cassirer, E.F., Cross, P.C., Plowright, R.K. \& Hudson, P.J. (2014). Costs and benefits of group living with disease: a case study of pneumonia in bighorn lambs (Ovis canadensis). Proc. Biol. Sci., 281, 20142331.

Manlove, K., Cassirer, E.F., Cross, P.C., Plowright, R.K. \& Hudson, P.J. (2016). Disease introduction is associated with a phase transition in bighorn sheep demographics. Ecology, 97, 2593-2602.
Marshall, T., Slate, J., Kruuk, L. \& Pemberton, J. (1998). Statistical confidence for likelihood-based paternity inference in natural populations. Mol. Ecol., 7, 639-655.

May, R.M. \& Anderson, R.M. (1987). COMMENTARY Transmission dynamics of HIV infection. Nature, 326, 137.

McAuliffe, L., Hatchell, F. \& Ayling, R. (2003). Detection ofMycoplasma. Vet. Rec., 153, 687-688.

Medley, G.F., Lindop, N.A., Edmunds, W.J. \& Nokes, D.J. (2001). Hepatitis-B virus endemicity: heterogeneity, catastrophic dynamics and control. Nat. Med., 7, 619-624.

Nussey, D., Coulson, T., Festa-Bianchet, M. \& Gaillard, J.M. (2008). Measuring senescence in wild animal populations: towards a longitudinal approach. Funct. Ecol., 22, 393-406.

Ott, J., Stevens, G., Groeger, J. \& Wiersma, S. (2012). Global epidemiology of hepatitis B virus infection: new estimates of agespecific HBsAg seroprevalence and endemicity. Vaccine, 30, 22122219.

Paterson, S. (1998). Evidence for balancing selection at the major histocompatibility complex in a free-living ruminant. J. Hered., 89, 289-294.

Paterson, S., Wilson, K. \& Pemberton, J. (1998a). Major histocompatibility complex variation associated with juvenile survival and parasite resistance in a large unmanaged ungulate population (Ovis aries L.). Proc. Natl Acad. Sci., 95, 3714-3719.

Paterson, S., Wilson, K. \& Pemberton, J.M. (1998b). Major histocompatibility complex variation associated with juvenile survival and parasite resistance in a large unmanaged ungulate population (Ovis aries L.). Proc. Natl Acad. Sci. USA, 95, 3714-3719.

Plowright, R.K., Manlove, K., Cassirer, E.F., Cross, P.C., Besser, T.E. \& Hudson, P.J. (2013). Use of exposure history to identify patterns of immunity to pneumonia in bighorn sheep (Ovis canadensis). PLoS ONE, 8, e61919.

Plowright, R.K., Peel, A.J., Streicker, D.G., Gilbert, A.T., McCallum, H., Wood, J. et al. (2016). Transmission or within-host dynamics driving pulses of zoonotic viruses in reservoir-host populations. PLoS Negl. Trop. Dis., 10, e0004796.

Prager, K., Greig, D.J., Alt, D.P., Galloway, R.L., Hornsby, R.L., Palmer, L.J. et al. (2013). Asymptomatic and chronic carriage of Leptospira interrogans serovar Pomona in California sea lions (Zalophus californianus). Vet. Microbiol., 164, 177-183.

Raberg, L., Graham, A.L. \& Read, A.F. (2009). Decomposing health: tolerance and resistance to parasites in animals. Philos. Trans. R. Soc. Lond. B Biol. Sci., 364, 37-49.

Roy, B. \& Kirchner, J. (2000). Evolutionary dynamics of pathogen resistance and tolerance. Evolution, 54, 51-63.

Schwaiger, F.-W., Gostomski, D., Stear, M., Duncan, J., McKellar, Q., Epplen, J. et al. (1995). An ovine major histocompatibility complex DRB1 allele is associated with low faecal egg counts following natural, predominantly Ostertagia circumcincta infection. Int. J. Parasitol., 25, $815-822$.

Solana, R., Pawelec, G. \& Tarazona, R. (2006). Aging and innate immunity. Immunity, 24, 491-494.

Spencer, S.E., Besser, T.E., Cobbold, R.N. \& French, N.P. (2015). 'Super'or just 'above average'? Supershedders and the transmission of Escherichia coli O157: $\mathrm{H} 7$ among feedlot cattle. J. R. Soc. Interface, 12, 20150446.

Streicker, D.G., Fenton, A. \& Pedersen, A.B. (2013). Differential sources of host species heterogeneity influence the transmission and control of multihost parasites. Ecol. Lett., 16, 975-984.

Tarazona, R., Solana, R., Ouyang, Q. \& Pawelec, G. (2002). Basic biology and clinical impact of immunosenescence. Exp. Gerontol., 37, 183-189.

Turner, J.C. (1977). Cemental annulations as an age criterion in North American sheep. J. Wildl. Manag., 41, 211-217.

Vallejo, A.N. (2007). Immune remodeling: lessons from repertoire alterations during chronological aging and in immune-mediated disease. Trends Mol. Med., 13, 94-102. 
VanderWaal, K.L. \& Ezenwa, V.O. (2016). Heterogeneity in pathogen transmission: mechanisms and methodology. Funct. Ecol., 30, 16061622.

Virgin, H.W., Wherry, E.J. \& Ahmed, R. (2009). Redefining chronic viral infection. Cell, 138, 30-50.

Waits, L.P. \& Paetkau, D. (2005). Noninvasive genetic sampling tools for wildlife biologists: a review of applications and recommendations for accurate data collection. J. Wildl. Manage., 69, 1419-1433.

Wertheim, H.F., Melles, D.C., Vos, M.C., van Leeuwen, W., van Belkum, A., Verbrugh, H.A. et al. (2005). The role of nasal carriage in Staphylococcus aureus infections. Lancet Infect. Dis., 5, 751-762.

Wigginton, J.E., Cutler, D.J. \& Abecasis, G.R. (2005). A note on exact tests of Hardy-Weinberg equilibrium. Am. J. Hum. Genet., 76, 887-893.

Wilson, K., Bjørnstad, O., Dobson, A., Merler, S., Poglayen, G., Randolph, S. et al. (2002). Heterogeneities in macroparasite infections: patterns and processes. Ecol. Wildl. Dis., 44, 6-44.

Woolhouse, M.E., Dye, C., Etard, J.-F., Smith, T., Charlwood, J. \& Garnett, G., et al. (1997). Heterogeneities in the transmission of infectious agents: implications for the design of control programs. Proc. Natl Acad. Sci., 94, 338-342.

Yagupsky, P. (2015). Kingella kingae: carriage, transmission, and disease. Clin. Microbiol. Rev., 28, 54-79.
Young, D., Hussell, T. \& Dougan, G. (2002). Chronic bacterial infections: living with unwanted guests. Nat. Immunol., 3, 1026 1032.

Ziegler, J.C., Lahmers, K.K., Barrington, G.M., Parish, S.M., Kilzer, K., Baker, K. et al. (2014). Safety and immunogenicity of a Mycoplasma ovipneumoniae Bacterin for domestic sheep (Ovis aries). PLOS ONE, 9, e95698.

\section{SUPPORTING INFORMATION}

Additional Supporting Information may be found online in the supporting information tab for this article.

\section{Editor, Giulio De Leo}

Manuscript received 16 March 2017

First decision made 23 April 2017

Second decision made 14 July 2017

Manuscript accepted 23 July 2017 